\title{
Qualidade de Vida e Sobrecarga de Cuidadores de Crianças com Câncer
}

Quality of Life and Work Overload of Caretakers of Children with Cancer

Calidad de Vida y Sobrecarga de Cuidadores de Niños con Câncer

Samkya F. de O. Andrade, Railda

F. Alves, Myriam de O. Melo \&

Maria J. Rodrigues

Universidade Federal da Paraíba

http://dx.doi.org/10.1590/1982 - 370002332013

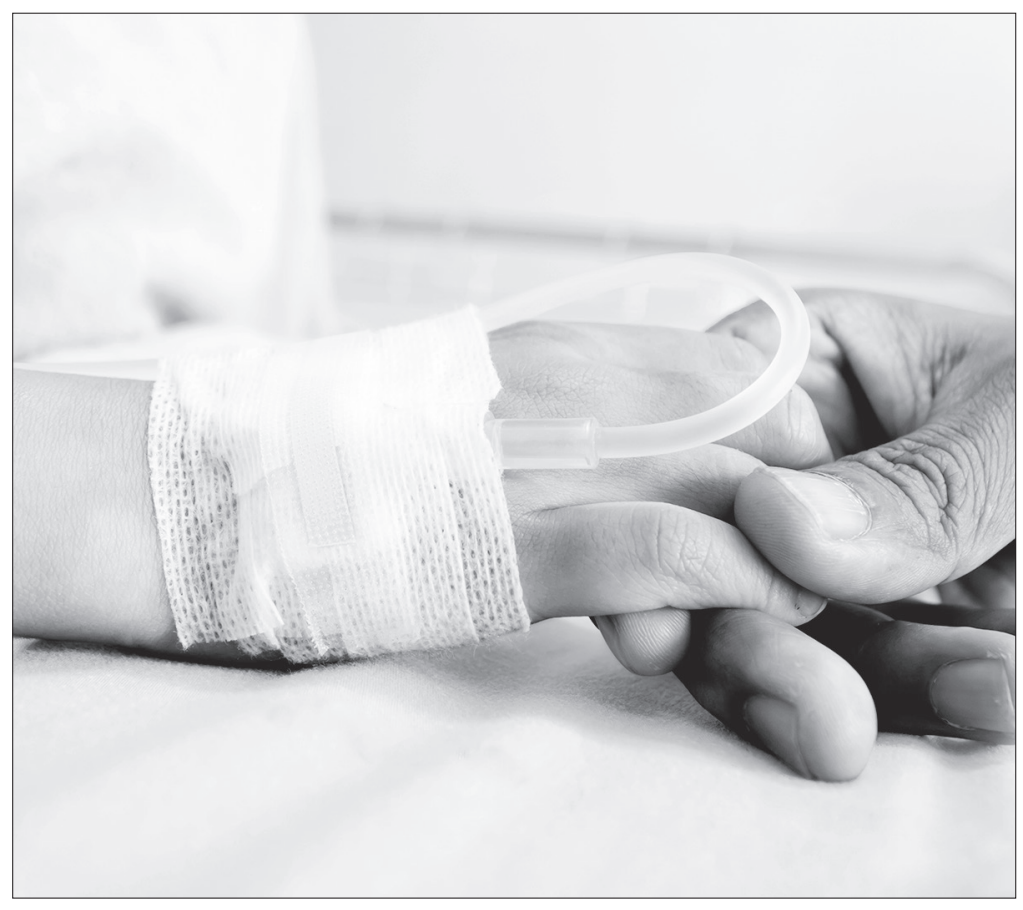


Resumo: O objetivo do estudo foi verificar a relação entre a qualidade de vida e a sobrecarga em cuidadores de crianças portadoras de neoplasia e assistidas em uma casa de apoio. A metodologia foi quantiqualitativa com a utilização de multimétodos de pesquisa. Os participantes $(\mathrm{N}=23)$ foram os cuidadores dos pacientes acompanhados pela Casa de Apoio à Criança com Câncer. Os instrumentos de coleta de dados foram: um questionário sociodemográfico, a Escala Whoqol - Bref, a Escala Zarit e uma entrevista por pautas. A análise dos dados se deu pela estatística descritiva e pela análise da enunciação. Os resultados apontam uma amostra essencialmente feminina (95,7\%); sendo $82,6 \%$ formada pelas mães. A maioria apresentou sobrecarga intensa $(54,5 \%)$. O domínio de maior prejuízo foi o psicológico $(53,98)$. As entrevistas confirmaram os dados obtidos nas escalas e mostraram mudanças significativas na vida dos cuidadores e da família, com prejuízo marcadamente para a vida social e a relação de trabalho. Os cuidadores sentem-se sobrecarregados, mas a sobrecarga permanece velada, já que a sua manifestação pode revelar emoções e sentimentos de culpa, difíceis de serem admitidos e elaborados.

Palavras-chave: Qualidade de Vida. Cuidadores. Câncer em crianças. Casa de apoio.

Abstract: This paper aims at verifying the relationship between the quality of life and work overload of caretakers of children with neoplasia that are cared for at a supporting home. The methodology was quantity-qualitative with use of multi methods of research. The participants $(\mathrm{N}=23)$ were the caretakers of patients cared for at the Support Home for Children with Cancer. The data collection instruments were: a socio-demographic questionnaire, the Whoqol - Bref Scale, the Zarit Scale, and one interview by topics. Data analysis was done by the descriptive statistics and by the enunciation analysis. The outcomes point to an essentially female sample $(95.7 \%)$; while mothers comprised $82.6 \%$. The majority presented intense overload (54.5\%). The predominant loss was psychological (53.98). The inerviews confirmed that obtained in the scaales and they showed significant changes in the life of caretakers and families, with marked losses for social life and work relations. The caretakers feel overloaded, but this overload remains disguised, as its manifestation may reveal emotions and guilty feelings, which are hard to be admitted and elaborated.

Keywords: Quality of Life; Overload; Caretgiver. Cancer in children. Support Home.

Resumen: El objetivo del estudio fue a verificar la relación entre la calidad de vida y la sobrecarga en cuidadores de niños portadores de neoplasia y asistidas en una casa de apoyo. La metodología fue cuanti-cualitativa con la utilización de multimétodos de pesquisa. Los participantes $(\mathrm{N}=23)$ fueron los cuidadores de los pacientes acompañados por la Casa de Apoyo a Niños con Cáncer. Los instrumentos de colecta de datos fueron: un cuestionario socio demográfico, la Escala Whoqol - Bref, la Escala Zarit y una entrevista por pautas. El análisis de los datos se dio por la estadística descriptiva y por el análisis de la enunciación. Los resultados apuntan una muestra esencialmente femenina (95,7\%); siendo un $82,6 \%$ formada por las madres. La mayoría presentó sobrecarga intensa $(54,5 \%)$. El dominio de mayor perjuicio fue el psicológico $(53,98)$. Las entrevistas confirmaron los datos obtenidos en las escalas y mostraron mudanzas significativas en la vida de los cuidadores y de la familia, con perjuicio marcadamente para la vida social y la relación de trabajo. Los cuidadores se sienten sobrecargados, pero la sobrecarga permanece velada, ya que su manifestación puede revelar emociones y sentimientos de culpa, difíciles de ser admitidos y elaborados.

Palabras-clave: Calidad de Vida; Sobrecarga; Cuidadores. Cancer en niños. Casa de apoyo. 
O interesse em estudar a qualidade de vida e a sobrecarga de cuidadores de crianças com câncer justifica-se, em primeiro lugar, porque a rotina de tal cuidador é um objeto de estudo privilegiado para se estudar a sobrecarga, já que o câncer, pelas suas características, é extenuante para o doente e para a pessoa que o cuida. Em segundo lugar, porque a manifestação dessa doença em criança gera grande mobilização da subjetividade (conflitos existenciais) da pessoa que cuida e de sua família como um todo.

O câncer funciona, muitas vezes, como um fator de desestruturação do meio familiar. Uma vez que a sua descoberta provoca sofrimento no cuidador e uma repentina alteração na rotina doméstica, como, por exemplo: acompanhar a criança em procedimentos agressivos e internações, aliado ao sentimento de impotência, medo da morte e possíveis recidivas (Nóbrega \& Pereira, 2011; Pereira, Magni, Mingione, \& Caran, 2009; Silva, Teles, \& Valle, 2005). Outro fator relevante é apontado por Beck e Lopes (2007) quando afirmam que a fragilização e o sofrimento causados pela doença tornam a criança mais dependente, o que exige da pessoa que cuida, em especial do cuidador principal, maior gasto de energia incidindo diretamente em perda de sua qualidade de vida.

A abordagem sobre a qualidade de vida e sobrecarga do cuidador põe de manifesto a dualidade de sentimentos vivida pelos sujeitos com relação, por um lado, à satisfação por cuidar do paciente e, por outro lado, ao sentimento de estarem sobrecarregados, uma vez que o câncer demanda cuidados específicos (Brito, Rezende, Malta, Schall, \& Modena, 2008; Sequeira, 2010). O cuidado resulta na adição de novas tarefas ao cotidiano e mudanças na esfera social e profissional do cuidador, o que pode influenciar diretamente na qualidade de vida dele
(Brito, Rezende, Malta, Schall, \& Modena, 2008; Sequeira, 2010).

O termo qualidade de vida (QV) apresenta dificuldade para ser definido de forma consensual. Hunt (1997) explica que tal dificuldade reside, em primeiro lugar, no fato de que existe concordância geral sobre o tema, ou seja, todos já sabem o que significa, sendo desnecessária uma definição formal. Em segundo lugar, não existe uma boa definição que abarque a complexidade do tema, ficando vulnerável e passível de ser aplicada a qualquer definição. McGuire (1991), corroborando essa afirmação, disse que o problema reside no fato de que cada um tem a sua ideia sobre QV.

A partir dos anos 90, estudiosos do tema (Pais-Ribeiro, 2009; Seidl \& Zannon, 2004) apresentaram um consenso quanto a dois aspectos relevantes do conceito de $\mathrm{QV}$, que são o fator multidimensionalidade, em que o construto deve considerar diferentes dimensões; e o fator subjetivo, que trata de considerar a percepção e avaliação pessoal do indivíduo em cada dimensão referente à QV. Seidl e Zannon (2004) apontam duas tendências no que se refere à conceituação do termo, uma como conceito genérico e outra relacionada à saúde. Os estudos que partem de concepção genérica incluem apenas pessoas saudáveis da população. Como referência dessa conceituação, tem-se a da Organização Mundial da Saúde (OMS) que afirma que QV é "a percepção do indivíduo de sua posição na vida, no contexto da cultura e sistema de valores nos quais ele vive e em relação aos seus objetivos, expectativas, padrões e preocupações" (Fleck, Louzada, Xavier, Chachamovich, Vieira, Santos, \& Pinzon, 2000, p. 179). Já o termo QV relacionado à saúde aponta para aspectos associados a doenças ou intervenções em saúde. 
Com relação à pessoa que cuida, a literatura mostra que há distinção entre o cuidador formal, caracterizado como o profissional de saúde que faz uma preparação acadêmica para tal; e o cuidador informal, que é o familiar ou amigo solicitado a fornecer cuidados ao paciente; e o cuidador social, que conforme a Classificação Brasileira de Ocupações (CBO) se refere àqueles que "cuidam de bebês, crianças, jovens, adultos e idosos, a partir de objetivos estabelecidos por instituições especializadas ou responsáveis diretos, zelando pelo bem-estar, saúde, alimentação, higiene pessoal, educação, cultura, recreação e lazer da pessoa assistida" (CBO, 2002, p. 607).

O ato de cuidar pode ser vivenciado com um significativo grau de dificuldade e sofrimento (Leite, 2006). A isso se atribui a questão da sobrecarga, denominada na literatura inglesa pelo termo "burden". O conceito diz respeito ao conjunto de consequências ocorridas após contato próximo do cuidador com o doente (Sequeira, 2010). Platt, em 1985, foi o primeiro estudioso a apresentar uma definição de sobrecarga, relacionando à presença de problemas ou dificuldades que afetam as vidas dos familiares cuidadores (Bocchi, 2004). Por envolver dimensões concretas e emocionais tal conceito diferencia-se em dois aspectos, o objetivo e o subjetivo. O objetivo se refere às consequências negativas observáveis no cuidador, resultantes do ato de cuidar, tais como mudanças na vida doméstica, relações sociais e familiares e na saúde física e psicológica (Albuquerque, 2010; Schene, 1990), além do impacto financeiro como ressalta Bandeira, Calzavara e Castro (2008). A sobrecarga subjetiva, por sua vez, consiste na percepção do familiar sobre a sua situação de cuidador, tais como a sensação de peso e de incômodo ao exercer determinadas funções (Bandeira, Calzavara, \& Castro, 2008; Sequeira, 2010).
Bandeira, Calzavara e Castro (2008) constataram em pesquisas internacionais que os familiares, apesar de se sentirem satisfeitos por cuidar do paciente, sentem-se, também, sobrecarregados, já que do cuidar resulta a adição de novas tarefas em seu cotidiano e mudanças na esfera social e profissional do cuidador. Com isso, a sobrecarga pode levar ao comprometimento da saúde física e psicológica do cuidador, acarretando em uma deterioração da qualidade de vida deste (Sequeira, 2010).

Apesar do crescente avanço de estudos nessa área, este estudo, ao se propor voltar seu foco de atenção aos cuidadores, parte do princípio de que a criança com neoplasia apresenta alto nível de dependência, o que acarreta o aumento da sobrecarga no cuidador, enfrentando, muitas vezes, situações de fadiga, depressão e insônia (Nóbrega \& Pereira, 2011).

O estudo aqui proposto teve como objetivo principal verificar a existência da relação entre a qualidade de vida e sobrecarga de cuidadores informais de pacientes pediátricos portadores de neoplasia. As metas para os objetivos específicos foram mensurar o nível de sobrecarga e qualidade de vida dos cuidadores; conhecer a percepção dos cuidadores acerca da sua qualidade de vida; compreender as concepções dos cuidadores acerca de como as atividades relacionadas ao cuidar Ihes sobrecarregam.

\section{Método}

A abordagem do estudo foi transversal e descritiva e o corpus captado e analisado mediante o uso de multimétodos de pesquisa. A amostra foi composta por 23 sujeitos e constituída, de forma não probabilística por conveniência, por cuidadores informais acompanhantes de crianças em tratamento quimioterápico ou radioterápico na Casa de 
Apoio à Criança com Câncer (CACC) e, eventualmente, no Hospital Universitário Alcides Carneiro (HU), com aqueles que acompanhavam os pacientes internos; ambos os locais situados em Campina Grande, Paraíba - Brasil. Foram incluídos os cuidadores familiares de pacientes pediátricos portadores de neoplasia, acompanhados pela CACC, seguindo o critério de aceitabilidade e disponibilidade. Foram excluídos os cuidadores que não se enquadravam na categoria de cuidadores informais. Todos assinaram o Termo de Consentimento Livre e Esclarecido (TCLE), após terem ciência dos objetivos e procedimentos da pesquisa.

\section{Instrumentos e Procedimentos}

Foram utilizados como instrumentos de coleta de dados um questionário sociodemográfico, a Escala de Qualidade de Vida Whoqol-Bref (Fleck et al. 2000), a Escala de Sobrecarga do Cuidador - Zarit (Sequeira, 2007) e uma entrevista por pautas (Gil, 1999). Todos aplicados pelas pesquisadoras para evitar a exclusão de participantes com baixa escolaridade e uniformizar a aplicação.

O questionário sociodemográfico (Rea \& Parker, 2000) coletou informações sobre: sexo, idade, profissão e situação profissional, além de questões relacionadas ao tempo de prestação do cuidado, à relação de parentesco e à divisão do cuidado.

A escala de qualidade de vida (Whoqol-Bref, 1998) é composta por 26 questões, sendo duas questões gerais de qualidade de vida e 24 referentes a cada uma das 24 facetas que compõem o instrumento original Whoqol100 (Fleck et al., 2000).

O instrumento avalia o domínio físico, como dor, sono, repouso e mobilidade; o domínio psicológico, como sentimentos positivos e autoestima; domínio das relações sociais, como suporte (apoio) social e atividade sexual; e o domínio referente ao meio ambiente, que diz respeito à segurança física, proteção e recursos financeiros.

A Escala de Sobrecarga do Cuidador (Zarit) é uma versão traduzida e adaptada por Sequeira (2007) a partir da Burden Interview Scale (Zarit \& Zarit, 1983; Marin, 1996; Scazufca, 2002). É um instrumento que permite avaliar a sobrecarga objetiva e subjetiva do cuidador informal e que inclui informações sobre saúde, vida social, vida pessoal, situação financeira, emocional e tipo de relacionamento.

A entrevista por pautas (Minayo, 2007) abarca tanto os conhecimentos relacionados com o tema proposto como favorece a interação social entre entrevistado e pesquisador. Apresenta certa estrutura (Gil, 1999) utilizada para explorar os itens ao longo da entrevista.

Os dados quantitativos foram analisados pela estatística descritiva e os qualitativos mediante a análise da enunciação proposta por Bardin (1977). Objetivando dar visibilidade aos dados, optou-se pelo uso da árvore de associação de sentidos, propostas por Spink e Frezza (1999).

A pesquisa foi cadastrada na Plataforma Brasil (0756.0.133.000-11) e seguiu as orientações éticas previstas pelas Diretrizes e Normas de Pesquisa em Seres Humanos, conforme a Resolução 466/2012 do ConseIho Nacional de Saúde.

\section{Resultados e discussões}

A amostra foi constituída por 23 participantes, cuja média de idade foi 35 anos $(d p=10,16)$, idade mínima 19 e máxima 65 anos, com predominância do sexo feminino $(95,7 \%)$, destes, $82,6 \%$ eram as mães. As profissões/ocupações apontam o predomínio de 30,4\% 
para agricultores e donas de casa não remuneradas, estando 47,8\% da amostra sem emprego. Referente à divisão do cuidado, 43,5\% são os únicos cuidadores da criança. Beck e Lopes (2007), discutindo a divisão de tarefas do cuidado entre os familiares, afirmam que mesmo que aconteça certa ajuda, a responsabilidade maior pelo cuidado fica a cargo do cuidador principal, principalmente nos casos de internação. Beck e Lopes (2007) discutem a importância do apoio dado aos cuidadores, enfatizando que os que recebem afeto e apoio têm menos probabilidade de se "despersonalizar", além de que a falta de suporte no cuidado desencadeia um esgotamento físico e mental.

Acerca do tempo de cuidado, 69,4\% dos entrevistados afirmaram prestar cuidados há menos de um ano. Pereira, Magni, Mingione e Caran (2009) asseguram que, mesmo o cuidado estando nos estágios iniciais e se configurando em pouco tempo, há indícios de que o início do tratamento e do diagnóstico acarretem mudanças significativas na rotina, além de gerar incertezas sobre a cura e o medo da morte.

\section{Análise da Escala Burden Interview (Zarit)}

Os dados a seguir, demonstrados na tabela 01, foram pontuados de acordo com as normas da adaptação para a população brasileira descrita por Scazufca (2002). Os resultados foram obtidos a partir da somatória das respostas dos entrevistados no instrumento, assim: o "nunca" era pontuado com 1; o "quase nunca" com 2; o "às vezes" com 3 e o "quase sempre" com 4.

Tabela 1. Níveis de Sobrecarga

\begin{tabular}{lcc}
\hline Níveis de sobrecarga & Frequência $(\mathrm{N})$ & Porcentagem $(\%)$ \\
\hline Inferior a 46 = Sem sobrecarga & 7 & 31.8 \\
Entre 46 e 56 = Sobrecarga ligeira & 4 & 13.6 \\
Superior a 56 = Sobrecarga intensa & 12 & $\mathbf{5 4 . 5}$ \\
Total & 23 & 100.0 \\
\hline
\end{tabular}

A tabela 01 mostra que apenas 31,8\% ( $\mathrm{N}=7)$ não apresentam nenhum nível de sobrecarga. Os outros níveis (sobrecarga ligeira e intensa), quando somados, indicam que 68,1\% ( $N=15)$ da amostra apresentam algum nível de sobrecarga. Assim, 13,6\% apresentam sobrecarga moderada, enquanto 54,5\% apresentam elevados níveis de sobrecarga. Garrido e Menezes (2004) afirmam que o grau de parentesco do cuidador está associado ao impacto, fazendo que os sujeitos da família nuclear apresentem maiores níveis de sobrecarga. No presente trabalho, 100\% dos entrevistados são familiares e $82,6 \%$ dos cuidadores são as mães dos pacientes. Silva, Barros e Hora (2011) afirmam que são as mães que assumem, na maioria dos casos, a função de cuidador do paciente pediátrico e estas, por sua vez, atribuem a si mesmas ou a elas é atribuído o papel de exclusividade do cuidado.

Na escala Burden Interview (Zarit), os quatro fatores apresentaram uma consistência interna satisfatória, medida pelo alfa de Cronbach, que indica a confiabilidade dos dados. Os fatores apresentaram os seguintes escores: Impacto de prestação de cuidados $(\alpha=0,93)$; Relação Interpessoal ( $\alpha=1)$; Expectativas ao cuidar $(\alpha=1)$; Percepção de autoeficácia $(\alpha=1)$. 
Os dados obtidos por meio da escala Zarit apresentam escores de sobrecarga em quase todos os fatores. Como a escala é composta de 5 alternativas (nunca $=1$; quase nunca $=2$; às vezes $=3$; muitas vezes $=4$ e quase sempre =5), a média dos itens é de 2,5. Sendo assim, os escores acima de 2,5 indicam sobrecarga.

A análise de tais fatores mostrou que os Fatores 1 (Impacto de prestação de cuidados), 3 (Expectativas ao cuidar) e 4 (Percepção de autoeficácia) apresentaram médias superiores a 2,5 $(2,52>2,5$ ( 3,447>2,5 / 3,32>2,5), com desvio padrão de 0,89 (Fator 1); $d p=1,07$ (Fator 3) e $d p=1,19$ (Fator 4). O único fator que apresentou escore menor que 2,5 foi o Fator 2 , referente à Relação Interpessoal $(1,55<2,5)$, com $d p=0,47$.

O fator 1, Impacto de prestação de cuidados $(2,52 d p=0,89)$, refere-se à sobrecarga com prestação de cuidados diretos, com destaque a alteração de saúde, o elevado número de cuidados, falta de tempo e desgaste físico e mental (Ricarte, 2009). Aqui, ainda que em níveis baixos, a sobrecarga é um fato, ao que Fernandes e Petean (2011) explicam que o baixo índice de sobrecarga se deve, muitas vezes, ao cuidado ser visto pelo cuidador familiar como gratificante ao invés de sobrecarregado e que os familiares, especialmente as mães, tendem a ter dificuldades a declarar que a criança afeta negativamente sua privacidade, saúde física e emocional e suas relações sociais.

O fator 2, Relação Interpessoal, avalia o impacto e as dificuldades inter-relacionais resultantes da prestação de cuidados (Ricarte, 2009). Tal fator foi o que apresentou menor escore de sobrecarga
$(1,55 d p=0,47)$, consonante com os achados por Ricarte (2009) e com os resultados referentes à qualidade de vida no domínio social. O baixo índice pode ser explicado, pelo fato de que $47,8 \%$ $(\mathrm{N}=11)$ dos entrevistados estavam internados, juntamente com suas crianças, e o restante acompanhado pela casa de apoio. Situação que favorece o contato com outros pacientes e cuidadores e diminui o isolamento, ainda que o relacionamento se limite aos outros cuidadores acompanhados pela casa e ao pessoal do hospital.

O fator 3, Expectativas ao cuidar, diz respeito às expectativas relacionadas com a prestação de cuidados, referente aos medos e receios por parte do cuidador (Ricarte, 2009). Este foi o que apresentou maior escore $(3,44 d p=$ 1,07). Tal preocupação é compreensível, quando se avalia o nível de envolvimento emocional na relação do cuidador familiar (Fernandes \& Petean, 2011).

O fator 4, Percepção de autoeficácia, relaciona à opinião do cuidador diante do seu desempenho enquanto prestador de cuidados (Ricarte, 2009). Tal fator foi o segundo com maior escore de sobrecarga $(3,32 d p=1,19)$. Fernandes e Petean (2011) afirmam que é compreensível, pelo fato de serem todos os cuidadores em questão familiares, condição que aumenta o envolvimento afetivo e a autocobrança em relação a que poderiam fazer sempre algo a mais.

\section{Análise da Escala de Qualidade de Vida - Whoqol-bref}

No Whoqol-Bref, os quatro domínios apresentaram consistência interna satisfatória, medida pelo alfa de Cronbach, que 
indica a confiabilidade dos dados. Os domínios apresentaram os seguintes escores: domínio físico $(\alpha=0,97)$; domínio psicológico $(\alpha=0,99)$; relação social $(\alpha=1)$; meio ambiente $(\alpha=0,97)$.

O domínio físico compreende a percepção sobre sua condição física; o domínio psicológico, a percepção sobre a condição afetiva e cognitiva; o domínio social avalia a percepção sobre os relacionamentos sociais e os papéis adotados na vida; e o domínio ambiental compreende a percepção sobre aspectos diversos relacionados ao ambiente em que vive (Nóbrega \& Pereira, 2011).

Ressalta-se que os resultados foram transformados em índices de 0 a 100, para melhor visualização, ou seja, o resultado mais próximo de 100 representa melhor qualidade de vida. Com isso, constatou-se que o domínio meio ambiente foi o melhor avaliado positivamente, com 77,58 ( $d p=14,85)$, seguido do domínio físico, com média de 66,30 $(d p=14,87)$. O domínio social, por sua vez, apresentou escore de 65,21 $(d p=16,02)$ e por último, o domínio psicológico, que apresentou escore de $53,98(d p=11,75)$. Fernandes e Petean (2011) sugerem que a satisfação positiva do domínio meio ambiente $(77,58 / d p=14,85)$ se deve ao fato de os demais prejuízos tomarem tamanha proporção que excedem as dificuldades relacionadas aos outros aspectos da vida do cuidador.

A boa avaliação do domínio físico $(66,30 d p=14,87)$ apresenta-se dissonante com a literatura especializada, uma vez que pesquisas apontam que os cuidadores apresentam problemas físicos com maior frequência do que a população geral. A explicação para tal resultado supõe-se que está correlacionada com a baixa média de idade dos participantes da pesquisa (35 anos/dp $=10,1)$ (Rubira, Marcon, Belasco, Gaíva, \& Espinosa, 2012).

Quanto ao domínio social, os escores de $65,21(d p=16,02)$ apontam satisfação. Resultado consoante com a pesquisa de Albuquerque, Silva, Ferreira, Costa Junior e Saldanha (2009) e Nóbrega e Pereira (2011) que explicam que o cuidador familiar considera a responsabilidade de cuidar como unicamente sua e, mesmo que receba pouco apoio social, o aceita como suficiente.

O domínio psicológico foi o que obteve menor pontuação $(53,98 d p=11,75)$, ao que Nóbrega e Pereira (2011) corroboram afirmando que os prejuízos psíquicos são maiores e mais intensos do que os físicos. Pimenta, Rodrigues e Greguol (2010) sugerem que os baixos índices para tal domínio podem estar relacionados ao abandono dos estudos e as atividades profissionais e de lazer, o que significa abandonar as atividades que mantêm a saúde psicológica do indivíduo. Prieto, Massa e Torres (2010) ressaltam o medo de possíveis recaídas e de comorbidades como fatores que agravam os prejuízos no domínio psicológico.

Objetivando dialogar com os dados obtidos na presente pesquisa, a tabela 2 sintetiza as correlações entre os domínios do Whoqol-bref e os fatores Zarit, incluindo algumas variáveis sociodemográficas. Verificou-se correlação negativa estaticamente significativa entre todos os domínios do Whoqol-bref e o fator Zarit impacto de prestação de cuidados, com destaque para o domínio social $(-0,619<0,01)$ com a soma Zarit. 
Tabela 2. Coeficientes de correlação de Sperman entre os domínios Whoqol-bref e as demais variáveis.

\section{Fatores Zarit}

\begin{tabular}{lcccccccc}
\hline $\begin{array}{l}\text { Domínios } \\
\text { WHOQOL-bref }\end{array}$ & Idade & Parentesco & $\begin{array}{c}\text { Divide } \\
\text { cuidado }\end{array}$ & Fator la & Fator Ilb & Fator Illc & Fator IV & Zarit \\
\hline Domínio Físico & $-0,008$ & 0,116 & 0,287 & $\mathbf{- 0 , 5 5 5 *}$ & $-0,342$ & $\mathbf{- 0 , 4 2 6}$ & 0,015 & $\mathbf{- 0 , 5 4 6}^{* *}$ \\
Domínio Psicológico & 0,286 & $-0,036$ & $-0,257$ & $\mathbf{- 0 , 4 5 5 *}$ & $-0,218$ & $-0,336$ & 0,153 & $\mathbf{- 0 , 4 5 5}^{* *}$ \\
Relações Sociais & 0,093 & 0,185 & 0,195 & $\mathbf{- 0 , 6 1 9 * *}$ & $-0,169$ & $-0,279$ & 0,042 & $\mathbf{- 0 , 4 7 0}^{* *}$ \\
Meio Ambiente & 0,258 & 0,175 & $-0,020$ & $\mathbf{- 0 , 4 9 0 *}$ & $-0,112$ & $-0,359$ & $\mathbf{0 , 4 6 6 *}$ & $\mathbf{- 0 , 4 3 1 ^ { * }}$ \\
Domínio Geral & $-0,019$ & $-0,113$ & 0,171 & $\mathbf{- 0 , 4 8 9 * *}$ & $-0,229$ & $-0,412$ & 0,201 & $\mathbf{- 0 , 5 2 0 *}$
\end{tabular}

Nota. a. Impacto de prestação de cuidados. b Relação interpessoal. c. Expectativas face ao cuidar. d. Percepção de autoeficácia; ${ }^{*} p<0,05 * * p<0,01$.

\section{Resultados e discussões das análises qualitativas}

Os dados demonstrados a seguir referem-se às análises de discursos obtidas nas entrevistas. A demonstração por meio das árvores de associação de sentidos (Spink \& Frezza, 1999), mostram, em linguagem coloquial, as falas dos sujeitos depois de analisadas e organizadas por categorias. Os resultados confirmaram os achados das escalas utilizadas neste estudo.

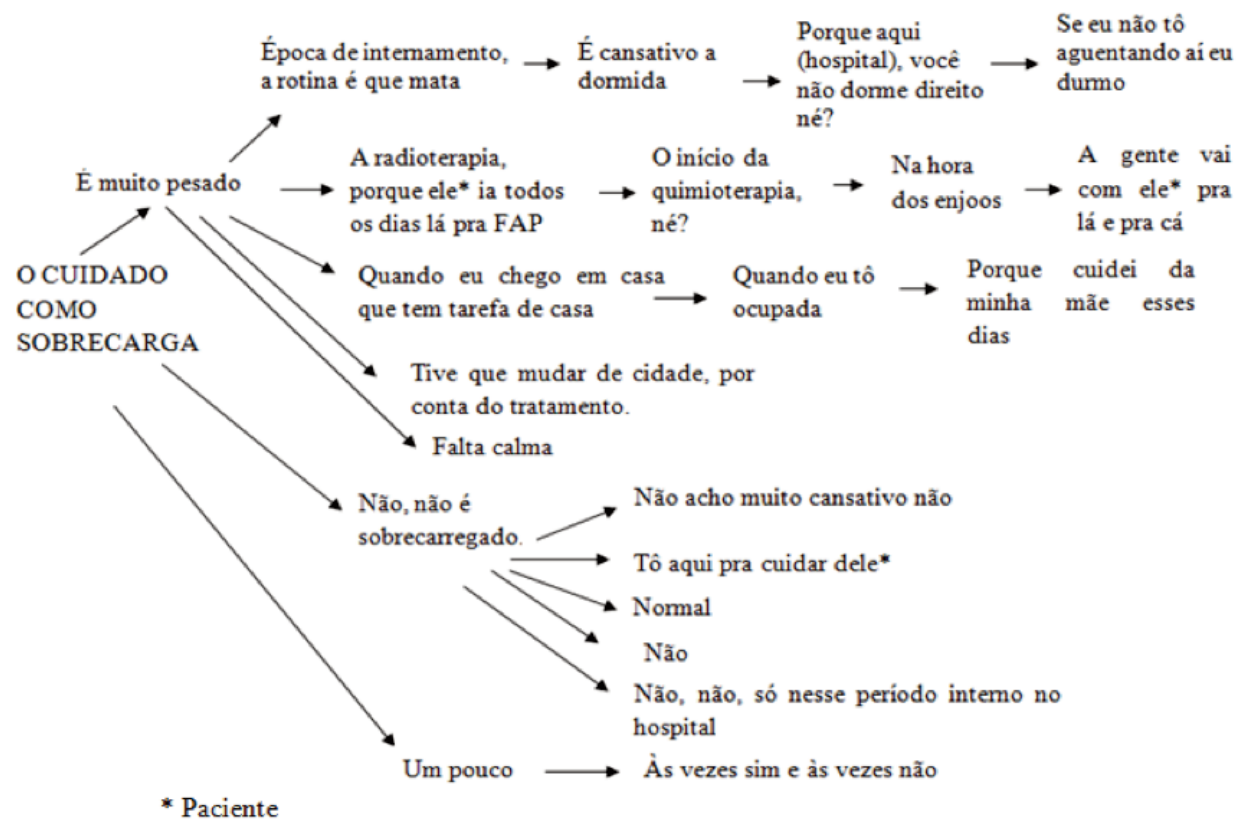

Figura 1. O cuidado como sobrecarga

A figura 1 mostra que os cuidadores se sentem mais sobrecarregados durante os períodos de internação, da radioterapia e da quimioterapia, porque é quando se recebe menos ajuda. Beck e Lopes (2007) afirmaram que a falta de ajuda no período de internação contribui para o aumento dos níveis de sobrecarga. Situação agravada pela limitação do convívio familiar, ficando a interação social restrita aos pacientes e a outros cuidadores internos. 
No período de quimioterapia e radioterapia, foram destacados, como fatores de sobrecarga, o cansaço de se locomover até o local de tratamento e as assistências aos efeitos colaterais. Araújo, Araújo, Souto e Oliveira (2009) discutem o comprometimento físico e emocional do cuidador especialmente nos chamados "momentos críticos", como uma provável cirurgia e os períodos de submissão à quimioterapia e radioterapia.

As atividades domésticas foram relatadas como sobrecarga, já que a amostra é composta por mulheres $(95,7 \%)$, as quais são responsáveis pelo cuidado ao doente bem como pelas atividades domésticas. O reconhecimento da sobrecarga ocorreu de modo ambivalente, pois ao reconhecerem-na, procediam imediatamente à negação ou minimização de seu efeito. Fernandes e Petean (2011) afirmam que embora o impacto exista, não pode ser reconhecido já que é difícil para um familiar, especialmente para a mãe, reconhecer que a criança seja um peso e o tempo a ela dispensado, sobrecarregado. Assim, a sobrecarga permanece velada porque a sua manifestação produz sentimentos ambivalentes, difíceis de serem admitidos e elaborados.

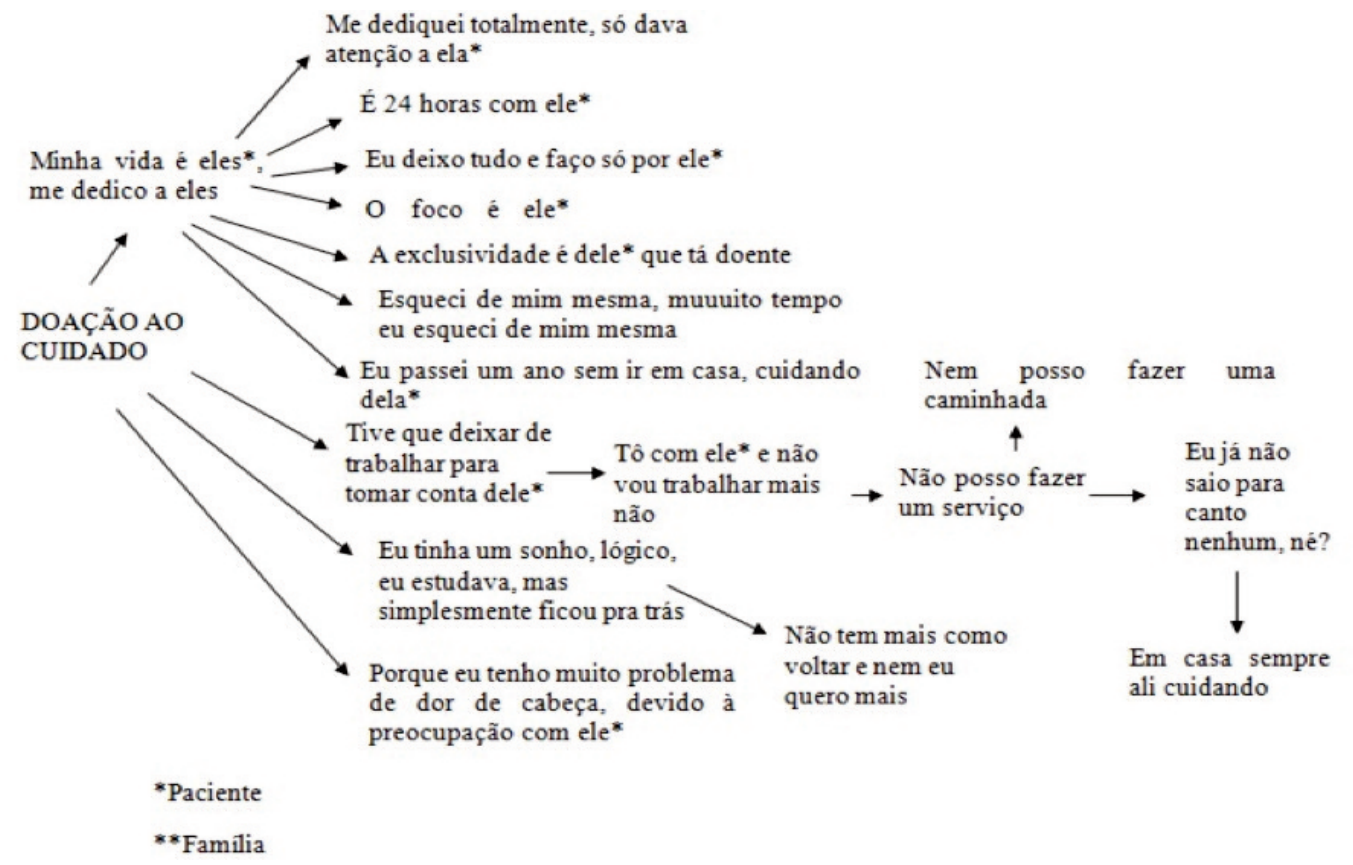

Figura 2 . Doação ao cuidado e mudanças do projeto de vida do cuidador

Na figura 2, os discursos apontam a quase total abdicação dos outros aspectos da vida do cuidador para dedicar-se totalmente ao doente. Fato explicado por ser a amostra formada basicamente $(82,6 \%)$ pelas mães dos pacientes, o que implica uma vivência de angústia e medo da morte do paciente, em alta intensidade. O desejo em diminuir o sofrimento do familiar e garantir que nada o prejudique faz com que o cuidador não meça esforços para ajudá-lo (Araújo, Morais, Lopes, Dantas, Oliveira, \& Maia, 2012). 


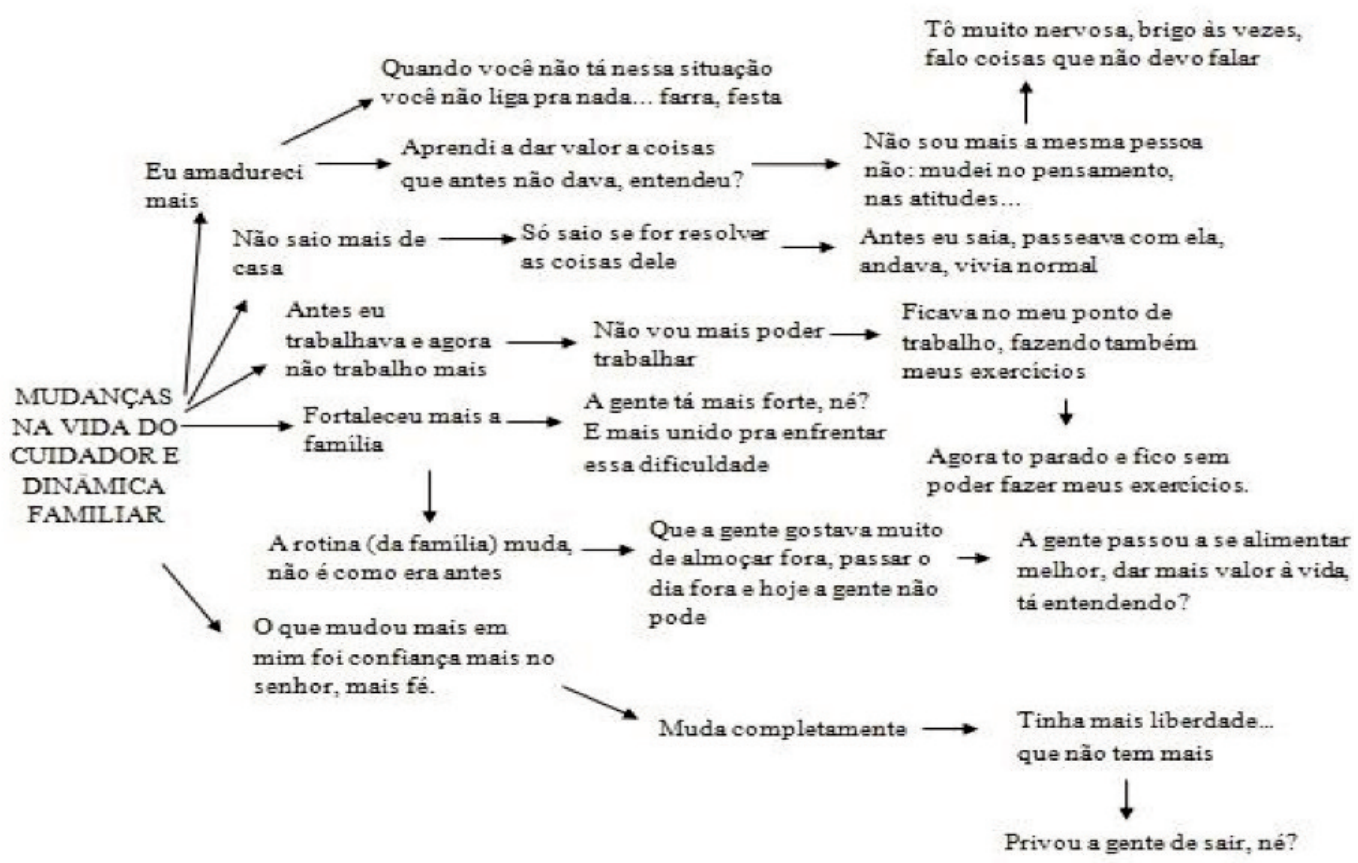

Figura 3. Mudanças após o diagnóstico na vida do cuidador e na dinâmica familiar

A figura 3 descreve as mudanças positivas e negativas observadas na vida dos cuidadores e na dinâmica familiar. Os interlocutores relataram que a família passou a ser mais unida, o sofrimento os deixou mais fortes e com visão diferente de como lidar com a vida. Kohlsdorf e Costa Junior (2008) explicam que ainda que a doença possa provocar conflitos e afastamento entre os membros da família, pode também provocar novos padrões de comportamento, como o aumento da coesão entre os familiares.

A rotina foi fortemente modificada. Isso ocorre porque na nova dinâmica familiar, a criança passa a ser o centro das atenções e a dinâmica familiar ajustada às suas necessidades. Com isso, ao cuidador incide considerável sobrecarga física e especialmente emocional, que culmina na diminuição das relações com outros membros da família (Grant, 2007). A privação das saídas de casa é citada como significante, uma vez que o contato social da criança fica limitado, devido à baixa da imunidade decorrente do tratamento (Beck \& Lopes, 2007). Alguns discursos mostraram que o cuidador abandonou por completo seus desejos e vontades. Faltar ao trabalho e deixar de trabalhar foram as queixas mais recorrentes. Nesta amostra, apenas $4,3 \%$ dos entrevistados estavam trabalhando. Muitos abandonaram ou conseguiram afastamento durante o período de internação. Dado corroborado por Mensorio, Kohlsdorf e Costa Junior (2009), que encontraram $76,6 \%$ de entrevistados que relataram modificação na sua rotina de trabalho. Kohlsdorf e Costa Junior (2008) encontraram $76,6 \%$ dos entrevistados confirmando a demissão, afastamento do emprego e necessidade de alteração do horário de trabalho. Situação que causa déficit na organização econômica da família em um momento em que as despesas aumentam em decorrência do tratamento (Beck \& Lopes, 2007; Kohlsdorf \& Costa Junior, 2008). As mudanças incluíram, ainda, o aumento da fé em Deus, além de atribuir a Ele a força para dar continuidade ao cuidado. Dado corroborado por Beck e Lopes (2007). 


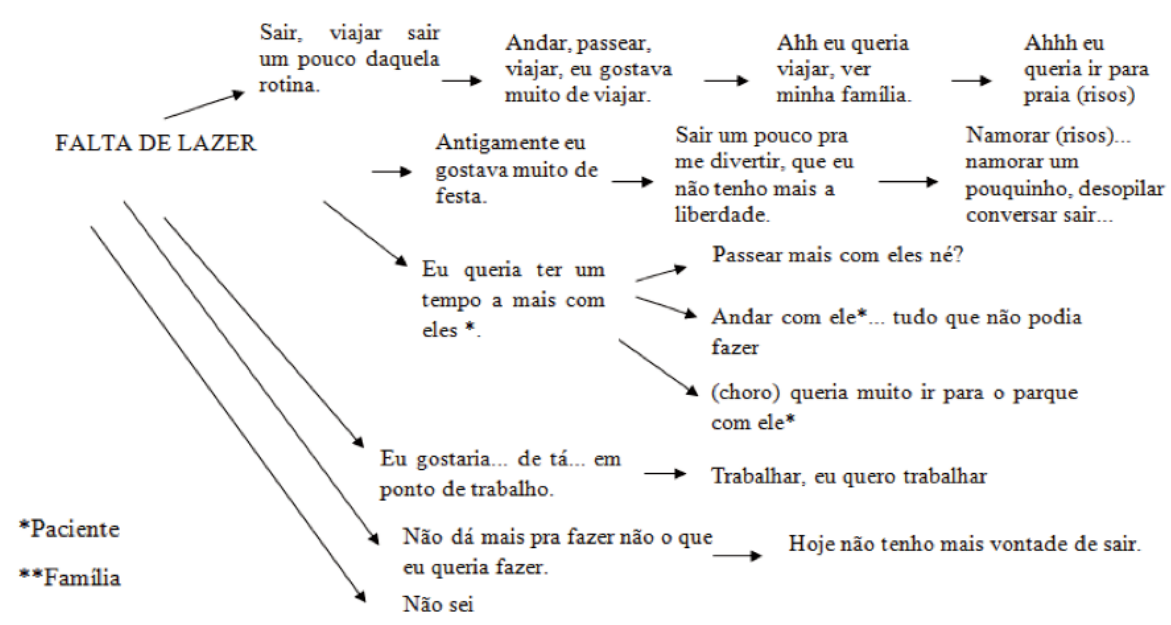

Figura 4. Falta de atividade de lazer

A figura 04 mostra as mudanças ocorridas na rotina do cuidador, destacando a falta de lazer relacionada diretamente ao aumento do estresse. Elenca as coisas que antes eram feitas e a impossibilidade de fazê-las na atualidade. Tal situação foi explicada como sendo resultado das limitações impostas pela situação do tratamento da pessoa cuidada.

As atividades de passeio, viagens e festas foram as mais comprometidas. Nos achados de pesquisa de Beck e Lopes (2007), as atividades de lazer sofriam prejuízos à medida que as internações se tornavam frequentes e as defesas imunológicas da criança impediam as saídas de casa e as visitas. Os discursos mais frequentes observados na figura 04 denotam a falta de lazer, tanto no plano pessoal como em relação às crianças, visto que estas ficam privadas do contato social em determinados períodos durante o tratamento. A dificuldade que têm os cuidadores em aceitar o lazer se justifica pelo sentimento de culpa, já que a renúncia, mesmo que temporária, das atividades de cuidado, para dedicar-se a uma atividade prazerosa, é intolerável (Araújo et al., 2012).

Os cuidadores disseram que gostariam de seguir trabalhando, mas as atividades de trabalho ficaram comprometidas em função das exigências do tempo dedicado ao cuidado.

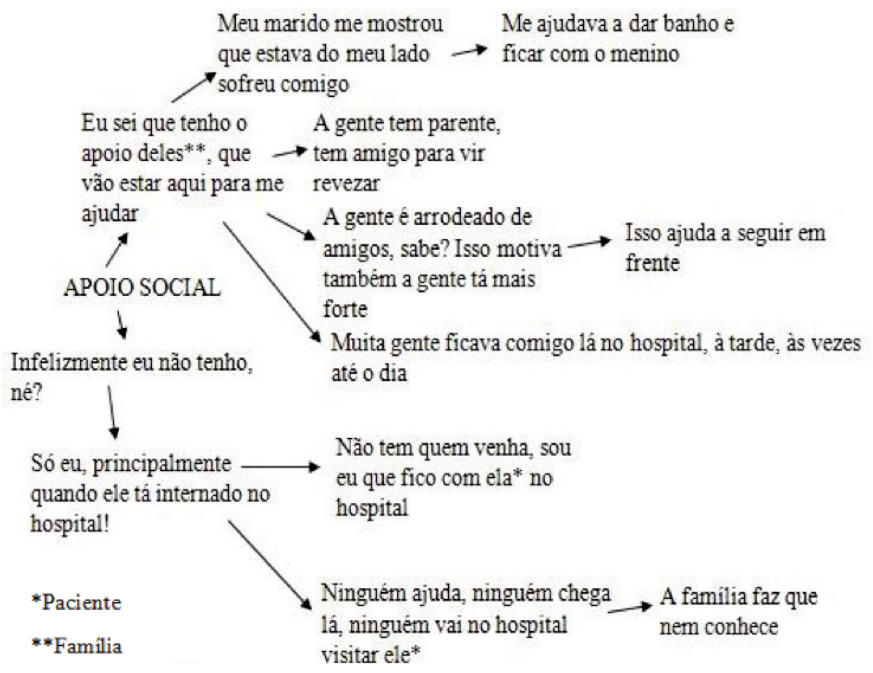

Figura 5. Apoio Social 
Sanchez, Ferreira, Dupas e Costa (2010) fazem a distinção entre rede social e apoio social. Rede social diz respeito à dimensão institucional ligada a um sujeito, como a vizinhança, igreja, sistema de saúde ou escola, enquanto que apoio social refere-se aos membros da rede social que são efetivamente importantes para a pessoa, envolvendo relações de troca e obrigações recíprocas, que podem contribuir positivamente para o estado de saúde das pessoas. Na figura 05, os sujeitos afirmaram que tinham apoio, em especial da família, durante o tratamento da criança. Percebeu-se que tal apoio possibilitava aos cuidadores suportar melhor a situação de sobrecarga resultante do cuidado, dado confirmado nos resultados da escala Zarit (Tabela 01) no Fator 2, referente à Relação Interpessoal $(1,55)$.

Aqueles que declararam não receber apoio referiram-se especialmente ao período da internação, quando o cuidado fica delegado integralmente ao cuidador principal. Esse resultado é consoante com os achados de Beck e Lopes (2007).

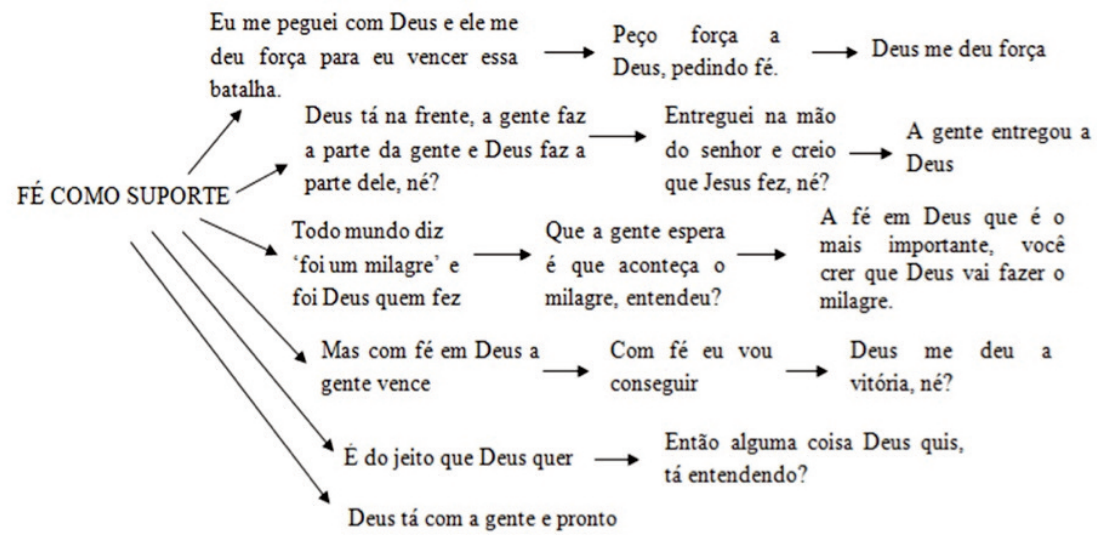

Figura 6. Fé como suporte

A figura 06 mostra que a fé é um importante suporte para o enfrentamento da doença do familiar e para a atuação do cuidador. Os entrevistados relataram um aumento da fé após o surgimento da doença e atribuíram a Deus, a força para suportar a situação atual. Outro discurso recorrente foi a submissão aos desígnios de Deus e o ideal de milagre e cura dos pacientes. Para Ramos (2007), os sentimentos angustiantes presentes após o diagnóstico de uma grave doença aumentam o otimismo em relação à cura e até em um milagre capaz de superar o prognóstico médico. A fé aponta para a diversificação das estratégias de enfrentamento da culpa e do estresse. Corroborando isso, Klassmann, Kochia, Furukawa, Higarashi e Marcon (2008) descreveram a experiência de mães cuidadoras de crianças com leucemia, que encontraram na fé alívio para o sofrimento, força para seguir cuidando e diminuição dos sentimentos de impotência e frustração.

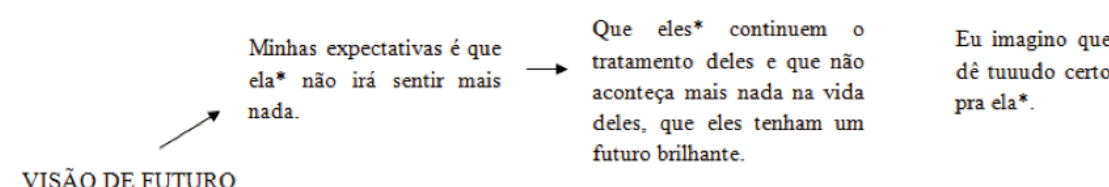

VISÃO DE FUTURO futuro brilhante.

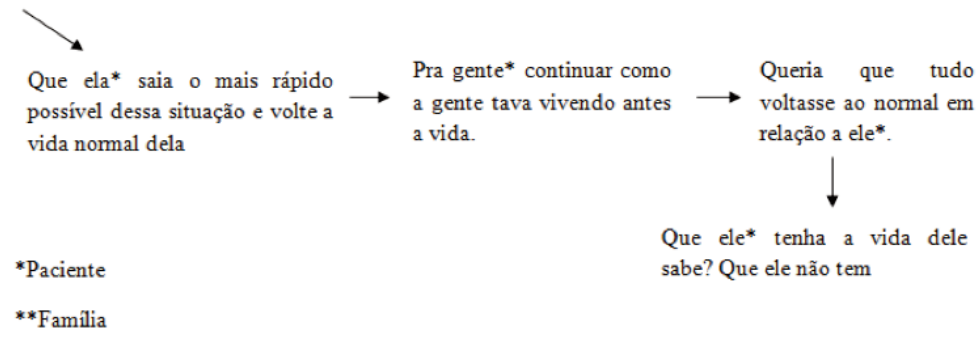

Figura 7. Visão de futuro 
Na figura 7, os discursos concentram-se na visão dos cuidadores acerca do futuro. Os dados deslindam a dificuldade dos entrevistados em voltar-se para o seu próprio amanhã em termos de projetos pessoais. O futuro pertence ao doente e foi representado pelo desejo de cura para o paciente como condição básica para a retomada do projeto pessoal e familiar. Assim, a cura é o projeto principal e só depois dela é que o paciente e a família poderão continuar ou, ao menos, tentar continuar os projetos de vida que deixaram para trás após o diagnóstico.

Tal conduta é facilmente compreendida, uma vez que o surgimento do câncer altera os projetos de toda a família, principalmente quando o principal sujeito atingido é uma criança, pois nela são depositados sonhos e desejos futuros. Com a doença, os sonhos e projetos são adiados e, muitas vezes, modificados (Beck \& Lopes, 2007).

\section{Considerações finais}

Esta investigação é importante porque seus resultados corroboram as poucas pesquisas voltadas ao estudo de cuidadores informais de pacientes infantis portadores de câncer. Confirmou-se que as mulheres/mães são as principais cuidadoras, uma vez que a prática do cuidado, em nossa sociedade, é fortemente ligada à muIher, muitas vezes entendida como prática extensiva às atividades domésticas (Melo, Rodrigues, \& Schmidt, 2009). Fato que sugere justificar também a vivência em alta intensidade dos sentimentos de angústia e medo da morte do paciente, tendo em vista que estão envolvidos fortes laços afetivos no ato do cuidado (Araújo et al. 2012). De modo que fica clara a relação positiva entre o grau de parentesco do cuidador e os maiores escores de sobrecarga (Garrido \& Menezes, 2004).

Quanto aos Fatores da Escala Burden Interview (Zarit), verificou-se que os maiores es- cores foram os fatores referentes a "Expectativas ao cuidar" e o fator "Percepção de autoeficácia". Resultado compreensível, já que os cuidadores em questão são os familiares, situação em que o envolvimento afetivo e a cobrança de si mesmo em fazer sempre algo mais afetam inevitavelmente tal percepção.

Quanto aos domínios da Escala de Qualidade de Vida (Whoqol-Bref), verificou-se o maior prejuízo no domínio psicológico, dado corroborado por Nóbrega e Pereira (2011) que afirmam o prejuízo psíquico como maiores e mais intensos do que os físicos.

A análise qualitativa mostrou que as atividades de cuidado provocam grandes mudanças e atingem significativamente a rotina dos cuidadores com prejuízo para a vida social e o trabalho. Situação que acarreta uma diminuição do cuidado pessoal, decorrente do grande número de atividades a realizar (Beck \& Lopes, 2007). Dados indicativos de sobrecarga e consequente perda da qualidade de vida.

Como limitação deste estudo, e sem deixar de considerar a relevância do material aqui suscitado, reconhece-se o número de participantes como fator que indica a necessidade do desenvolvimento de novos estudos na área, com diferentes delineamentos, visando à ampliação do entendimento do fenômeno.

Por fim, espera-se, com este estudo, contribuir ao enriquecimento da literatura, tendo em vista a escassez de material sobre este tema, uma vez que a grande maioria das pesquisas acerca da sobrecarga de cuidadores volta-se para área geriátrica ou psiquiátrica. Espera-se, também a criação de políticas de apoio mais efetivo aos cuidadores que, juntamente com o paciente, padecem, além do câncer, de um sofrimento que se expande para as esferas física, psicológica e social. 


\section{Samkya F. de O. Andrade}

Mestranda pela Universidade Federal da Paraíba, João Pessoa - PB. Brasil.

E-mail: samkyafernandes@ hotmail.com

\section{Railda F. Alves}

Universidade Federal da Paraíba, João Pessoa - PB. Brasil.

E-mail: raildafernandesalves@ gmail.com

\section{Myriam de O. Melo}

Graduada em Psicologia pela Universidade Federal da Paraíba, João Pessoa - PB. Brasil

E-mail: myriamoliveiramelo@ hotmail.com

\section{Maria J. Rodrigues}

Graduada em Psicologia pela Universidade Federal da Paraíba, João Pessoa - PB. Brasil

Endereço para envio de correspondência:

Rua Libia Cardoso da Silva, 79, Monte Santo. CEP: 58400-808. Campina Grande - PB. Brasil

Recebido 12/10/2013, $1^{\text {a }}$ Reformulação 09/08/2014, Aprovado 09/12/2014. 
Albuquerque, E. P. T. de. (2010). Sobrecarga de Familiares de Pacientes Psiquiátricos: Estudo de Diferentes Tipos de Cuidadores. Mestrado em Psicologia, Universidade Federal do São João del-Rei.

Albuquerque, J. R. de, Silva, J. da., Ferreira, F. A. R., Costa Junior, C. P. da, \& Saldanha, A. A. W. (2009). Resiliência e Qualidade de Vida de Mães de Criança com Câncer. Trabalho apresentado no 15 Encontro Nacional da Abrapso. Maceió.

Araújo, L. Z. S. de, Araújo, C. Z. S. de, Souto, A. K. de B. A., \& Oliveira, M. da S. (2009). Cuidador principal de paciente oncológico fora de possibilidade de cura, repercussões deste encargo. Revista Brasileira de Enfermagem, 62(1), 32-37. doi: 10.1590/S0034-71672009000100005.

Araújo, P. C. B. de, Morais, D., Lopes, S. S. de M., Dantas, M. M. C., Oliveira, L. C. B. de., \& Maia, E. M. C. (2012). Estratégias de enfrentamento utilizadas por cuidadores de crianças com câncer. Trabalho apresentado no 13 Congresso Virtual de Psiquiatria.com.

Bandeira, M., Calzavara, M. G. P., \& Castro, I. (2008). Estudo de validade da escala de sobrecarga de familiares cuidadores de pacientes psiquiátricos. Jornal Brasileiro de Psiquiatria, 57(2), 98-104. doi: 10.1590/S0047-20852008000200003.

Bardin, L. (1977). Análise de conteúdo. Lisboa, PT: Edições 70.

Beck, A. R. M., \& Lopes, M. H. B. de M. (2007) Cuidadores de crianças com câncer: aspectos da vida afetados pela atividade de cuidador. Revista Brasileira de Enfermagem, 60, 670-675. doi: 10.1590/S0034-71672007000600010.

Bocchi, S. C. M. (2004). Vivenciando a sobrecarga ao vir-a-ser um cuidador familiar de pessoa com acidente vascular cerebral (avc): uma análise do conhecimento. Revista Latino-Americana de Enfermagem, 12, 115-121. doi: 10.1590/S0104-11692004000100016.

Brito, V. F. D. S. de, Rezende, A. M., Malta, J. D., Schall, V. T., \& Modena, C. M. (2008). Oficinas para cuidadores de crianças com câncer: uma proposta humanizada em educação em saúde. Psicologia Hospitalar. 6(1), 66-81. Recuperado de http://pepsic.bvsalud.org/scielo.php?pid=S1677-74092008 000100006\&script $=$ sci_arttext

Classificação Brasileira de Ocupações CBO. (2002). Estrutura, tábua de conversão e índice de títulos. (Vol. 3, pp. 1215). Brasília, DF: MTE, SPPE

Fernandes, A. C. P., \& Petean, E. B. L. (2011). Sobrecarga Emocional e Qualidade de Vida em Mães de Crianças com Erros Inatos do Metabolismo. Psicologia: Teoria e Pesquisa, 27(4), 459-465. doi: 10.1590/S0102-37722011000400009.

Fleck, M. P. A., Louzada, S., Xavier, M., Chachamovich, E., Vieira, G., Santos, L., \& Pinzon, V. (2000). Aplicação da versão em português do instrumento abreviado de avaliação da qualidade de vida "WHOQOL-bref". Revista de Saúde Pública, 34, 178-183. doi: 10.1590/S003489102000000200012.

Garrido, R., \& Menezes, P. R. (2004). Impacto em cuidadores de idosos com demência atendidos em um serviço psicogeriátrico. Revista de Saúde Pública, 38, 835-841. doi: 10.1590/S003489102004000600012 .

Gil, A. C. (1999). Métodos e técnicas de pesquisa social. São Paulo: Atlas.

Grant, C. H. Vivências de Cuidadores de Crianças e adolescentes com câncer: Uma Reflexão sobre o Apoio Psicológico. (2007). Monografia de Psicologia. Centro Universitário Franciscano, Rio Grande do Sul.

Hunt, S. (1997). Defining quality of life: The practical Importance of conceptual Clarity - Technical, Ethical, and Interpretative Issues. Monitor MOT, 2, 9-12.

Klassmann, J., Kochia, K. R. A., Frukawa, T. S., Higarashi, I. H., \& Marcon, S. S. (2008). Experiência de mães de crianças com leucemia: sentimentos acerca do cuidado domiciliar. Revista da Escola de Enfermagem. São Paulo, 42(2), 321-330. doi $10.1590 / \mathrm{S} 0080$ 62342008000200016

Kohlsdorf, M., \& Costa Junior, A. L. da. (2008). Estratégias de enfrentamento de 
pais de crianças em tratamento de câncer. Estudos de Psicologia, Campinas, 25(3), 417-429. doi: 10.1590/S0103166X2008000300010

Leite, M. M. (2006). Impacto da demência de Alzheimer no cônjuge prestador de cuidados. Tese de doutorado, Faculdade de Psicologia e de Ciências da Educação, Porto.

Marín, M. (1996). Adaptación para nuestro medio de la escala de sobrecarga del cuidador de Zarit. Revista Multidisciplinar de Gerontologia, 6(4), 338-346.

Mcguire, A. M. (1991). Quality of life in women with epilepsy. In M. R. Trimble. (Ed). Women and epilepsy. Chichester: John Wiley \& Sons.

Mensorio, M. S., Kohlsdorf, M., \& Costa Junior, Á. L. (2009). Cuidadores de crianças e adolescentes com leucemia: análise de estratégias de enfrentamento. Psicologia em Revista, 15(1), 158-176. doi: $\quad 10.5752 /$ P.16789523.2009V15N1P158.

Minayo, M. C. S. (2007). Trabalho de campo: contexto de observação, interação e descoberta. In Anais do Congresso Nacional de Saúde do adolescente, pesquisa social: Teoria, método e criatividade. 26. Petropólis, RJ: Editora Vozes.

Nóbrega, K. I. M. da., \& Pereira, C. U. (2011). Qualidade de vida, ansiedade e depressão em cuidadores de crianças com neoplasia cerebral. Psicologia: Teoria e Prática, 13(1), 48-61. Recuperado de http://pepsic.bvsalud.org/scielo.php?pid= $\begin{array}{llllll}\mathrm{S} & 1 & 5 & 1 & 6\end{array}$ $36872011000100004 \&$ script $=$ sci_arttext

Pais-Ribeiro, J. (2009). A importância da qualidade de vida para a psicologia da saúde. In: Cruz, J. P., Jesus, S. N. \& Nunes, C. (Cords.), Bem-Estar e Qualidade de Vida (pp. 31-49). Alcochete: Textiverso.

Pereira, M. S., Magni, R. H. H., Mingione, I. C. D., \& Caran, E. M. M. (2009). Os desafios diários do cuidador de crianças e de adolescentes com câncer. Prática Hospitalar, 11(62), 51-53.

Pimenta, R. de A., Rodrigues, L. A., \& Greguol, M. (2010). Avaliação da Qualidade de Vida e Sobrecarga de Cuidadores de Pessoas com Deficiência Intelectual. Revista Brasileira de Ciências da Saúde, 14(3), 69-76. doi: 10.4034/RBCS.2010.14.03.10

Prieto, A. M., Massa, E. R., \& Torres, I. E. F. (2011). Percepción de la calidad de vida de cuidadores de niños com cardiopatia congénita Cartagena, Colombia. Invest Educ Enferm. 29(1, 9-18. Recuperado de file://C:/Users/cliente/Downloads/Dialnet-PercepcionDeLaCalidadDeVidaDeCuidadoresDeNinosConC-3730758.pdf

Ramos, S. E. B. (2007). Os familiares cuidadores da criança com doença de mau prognóstico: Fundamentos para uma intervenção no âmbito dos cuidados paliativos. Dissertação de mestrado em Cuidados Paliativos, Universidade de Lisboa.

Rea, I. M. \& Parker, R. A. (2000). Metodologia de pesquisa: do planejamento à execução. São Paulo: Pioneira.

Ricarte, L. F. C. S. (2009). Sobrecarga do cuidador informal de idosos dependentes no Concelho da Ribeira Grande. Dissertação de mestrado em Ciências da Enfermagem. Instituto de Ciências Biomédicas Abel Salazar, Universidade do Porto, Portugal.

Rubira, E. A.; Marcon, S. R.; Belasco, A. G. S.; Gaíva, M. A. M \& Espinosa, M. M. (2012). Sobrecarga e qualidade de vida de cuidadores de criança e adolescentes com câncer em tratamento quimioterápico. Acta Paul Enferm., 25(4), 567-573. doi: 10.1590/S010321002012005000020

Sanchez, K. de O. L., Ferreira, N. M. L. A., Dupas, G. \& Costa, D. B. (2010). Apoio social à família do paciente com câncer: identificando caminhos e direções. Revista Brasileira de Enfermagem, 63(2), 290-299. doi:10.1590/S003471672010000200019 .

Silva, T. C. de O.; Barros, V. F. \& Hora, E. C. (2011). Experiência de ser um cuidador familiar no câncer infantil. Rev Rene, 12(3), 526-531. Recuperado de http://www.revistarene.ufc.br/revista/ind ex.php/revista/article/view/258/pdf 
Scazufca, M. (2002). Versão brasileira da escala Burden Interview para avaliação de sobrecarga em cuidadores de indivíduos com doenças mentais. Revista Brasileira de Psiquiatria, 24(1), 12-17. doi: 10.1590/S1516-44462002000100006.

Schene, A. H. (1990). Objective and subjective dimensions of family burden. Towards an integrative framework for research. Social Psychiatry and Psychiatric Epidemiology, 25, 289-297. doi: 10.1007/BF00782883

Seidl E. M. F \& Zannon C. M. L. C. (2004). Qualidade de vida e saúde: aspectos conceituais e metodológicos. Caderno de Saúde Pública, 20(2), 580-588. doi: 10.1590/S0102-311X2004000200027.

Sequeira, C. A. da C. (2010). Adaptação e validação da Escala de Sobrecarga do Cuidador de Zarit. Revista Referência, 2(12), 13-19. Recuperado de file://C:/Users/cliente/Downloads/1Revista_Refer\%C3\%AAncia.pdf
Sequeira, C. (2007). Cuidar de Idosos Dependentes. Coimbra, PT: Quarteto Editora.

Silva, G. M. da Teles, S. S. \& Valle, E. R. M. do. (2005). Estudo sobre as publicações brasileiras relacionadas a aspectos psicossociais do câncer infantil -período de 1998 a 2004. Revista Brasileira de Cancerologia, 51, 253-261.

Spink, M. J. \& Frezza, R. M. Rigor e visibilidade: a explicação dos passos da interpretação. In: M. J. Spink, (Org.), Práticas discursivas e produção de sentidos no cotidiano: aproximações teóricas e metodológicas. (pp. 93-122). São Paulo, SP: Cortez, 93-122.

Zarit, S. H. \& Zarit, J. M. (1983). The memory and behaviour problems checklist and the burden interview. Technical report. Pennsylvania State University. 
Na edição 34.4, no artigo "Qualidade deVida e Sobrecarga de Cuidadores de Crianças com Câncer" onde se lê: Samkya F. de O. Andrade; Myriam de O. Melo \& Maria J. Rodrigues - Universidade Federal da Paraíba e Railda F. Alves Universidad de Granad, UGR, Espanha, o correto seria: Samkya F. de O. Andrade; Railda F. Alves; Myriam de O. Melo \& Maria J. Rodrigues - Universidade Estadual da Paraíba. http://dx.doi.org/10.1590/1982-370002332013 